\title{
EMPIRICAL MODEL REDUCTION OF CONTROLLED NONLINEAR SYSTEMS
}

\author{
Sanjay Lall, Jerrold E. Marsden, Sonja Glavaški \\ Control and Dynamical Systems \\ California Institute of Technology \\ Pasadena, CA 91125, USA. \\ sanj@cds.caltech.edu
}

\begin{abstract}
In this paper we introduce a new method of model reduction for nonlinear systems with inputs and outputs. The method requires only standard matrix computations, and when applied to linear systems results in the usual balanced truncation. For nonlinear systems, the method makes used of the Karhunen-Loève decomposition of the state-space, and is an extension of the method of empirical eigenfunctions used in fluid dynamics. We show that the new method is equivalent to balanced-truncation in the linear case, and perform an example reduction for a nonlinear mechanical system.
\end{abstract}

Full length paper: http://www.cds.caltech.edu/ sanj/home/publications/ifac99_empmr

Keywords: Karhunen-Loève decomposition, balanced truncation, model reduction, principal component analysis, total least-squares, Hankel norm.

\section{Introduction}

One of the difficulties in designing controllers for complex physical systems is in the problem of modeling. Very often, we have mathematical models which are highly nonlinear partial differential equations, for which control design techniques are little understood.

An example is given by formation flight; accurate models are known for the fluid dynamics, and for the aircraft motion, which together can be accurately simulated. However, on paper, the equations of motion consist of partial and ordinary differential equations coupled via their boundary conditions, a formulation which offers little to the control designer. It is therefore a problem of considerable interest to construct explicit low-order models.

The key difference between modeling for control, and modeling for analysis of dynamical system behavior is that in the latter case the system behavior is autonomous, with no external inputs driving the system. However, in the former case of controlled systems, we are concerned with preserving the relationship between the system behavior and the system inputs and outputs, or actuators and sensors. Linear input-output systems enjoy several model reduction techniques, including modal truncation, balanced truncation, and optimal Hankel, $H_{\infty}$ and $H_{2}$ norm methods.

One method which has been used extensively for dynamical systems analysis is the Karhunen-Loève decomposition, in particular in the construction of models for complex fluid flows [6]. In this paper, we extend this procedure to show how it may be used for con- struction of explicit, low order nonlinear models for complex controlled nonlinear systems. The low order model is constructed in such a way as to preserve important features of the input-output behavior, so that it may be used for control design.

The central idea of applying the Karhunen-Loève decomposition for model reduction is to search for a low dimensional affine subspace of the state space, in which the attractor is contained. In general a subspace of low (or finite) dimension may not exist, and the decomposition provides a way of finding the subspace of given dimension which best approximates that containing the attractor. Once such a subspace is found, a Galerkin projection can be applied to project the dynamics onto it, so that the high-dimensional system is approximated by a small number of nonlinear ordinary differential equations.

Once an appropriate measure is assigned to the space of trajectories of the system, the problem of finding the subspace can be formulated as a 2-norm optimization problem. This has the advantage of requiring only linear matrix computations, despite its usefulness in application to nonlinear systems. The optimization need not be performed analytically; instead data from experiment or simulation can be used directly.

The model reduction method we describe in this paper combines the features of the Karhunen-Loève decomposition and balanced-truncation. This provides an immediate computable procedure for model reduction of nonlinear input-output systems, requiring only linear matrix computations. For autonomous systems, it corresponds to finding the subspace which 
best approximates the attractor of the dynamical system. When applied to linear input-output systems, it results in the usual balanced truncation of the system, with the accompanying a-priori error bounds in the $H_{\infty}$ and Hankel norms.

We conclude the paper with an example to illustrate the application of the method to a nonlinear mechanical system.

\section{Model reduction for autonomous systems}

In this section we give an outline of existing techniques using the Karhunen-Loève decomposition for model reduction of nonlinear autonomous uncontrolled systems of the form

$$
\dot{x}(t)=f(x(t))
$$

where $x(t) \in \mathbb{R}^{n}$. This procedure was introduced for the analysis of turbulence by Lumley [8].

The method makes essential use of empirical data, taken either from experiments or from numerical simulation, consisting of sampled measurements $\left\{x^{(1)}, \ldots, x^{(N)}\right\}$ of $x(t)$. The next step is to perform a principal component analysis of this data, to find how well it may be approximated by projection onto a $k$-dimensional subspace of the original $n$-dimensional state space.

The Karhunen-Loève decomposition. The KarhunenLoève decomposition provides a method for finding this best approximating subspace. We give here a brief outline of the method, which goes back to Karl Pearson [10]; for details see [6].

We can characterize the subspace $S \subset \mathbb{R}^{n}$ by the projection operator $Q$ mapping $\mathbb{R}^{n}$ onto $S$. We would like to find $Q$ to minimize

$$
H(Q)=\sum_{i=1}^{N}\left\|x^{(i)}-Q x^{(i)}\right\|_{2}^{2}
$$

the total squared perpendicular distance of the points from the $k$-plane. The following result is standard.

Theorem 1. (Total Least Squares) Let $R$ be the correlation matrix of the data, defined by

$$
R:=\sum_{i=1}^{N} x^{(i)} x^{(i) *}
$$

and let $\lambda_{1} \geq \lambda_{2} \geq \cdots \lambda_{n}$ be the ordered eigenvalues of $R$. Then

$$
\min _{Q} H(Q)=\sum_{i=n-k+1}^{n} \lambda_{i}
$$

where the minimum is over all rank $k$ projections $Q$.

In general, $R$ may not have rank $n$, if the given data lies within a strict subspace of $\mathbb{R}^{n}$. Let $s=\operatorname{rank} R$, and let $\phi_{1}, \phi_{2}, \ldots, \phi_{s}$ be orthonormal eigenvectors of $R$, corresponding to the nonzero $\lambda_{i}$. Each $x^{(i)}$ can be written as

$$
x^{(i)}=\sum_{j=1}^{s} a_{i j} \phi_{j}
$$

where $a_{i j}=\left\langle x^{(i)}, \phi_{j}\right\rangle$, and $\left\langle\phi_{i}, \phi_{j}\right\rangle=\delta_{i j}$. The optimal $k$-dimensional subspace approximant is given by

$$
\hat{x}^{(i)}=\sum_{j=1}^{k} a_{i j} \phi_{j}
$$

Denote by $P$ the $k \times n$ matrix whose rows are $\phi_{1}, \ldots, \phi_{k}$, so that $P P^{*}=I$. The projected approximant to $x$ is given by $P^{*} P x \in S$, and $y=P x$ is a representation in terms of the new coordinates $\phi_{i}$ on $S$. This subspace approximant is then optimal, in the sense that the total energy (2-norm) in the subspace is given by

$$
\sum_{i=1}^{N}\left\|P x^{(i)}\right\|_{2}^{2}=\sum_{j=1}^{k} \lambda_{j}
$$

and this is the maximum achievable by any $k$-plane.

Model reduction. The above procedure finds the optimal subspace; in order to allow affine variation we make use of the fact that the optimal affine subspace passes through the mean of the data. Hence, we construct the correlation matrix as

$$
R_{i j}=\sum_{p=1}^{N}\left(x_{i}^{(p)}-\bar{x}_{i}\right)\left(x_{j}^{(p)}-\bar{x}_{j}\right)
$$

where $\bar{x}=\frac{1}{N} \sum_{i=1}^{N} x^{(i)}$ is the mean of the data. The eigenvalues of $R$ now provide us with information as to how close an approximation of the data is provided by a $k$-dimensional subspace; the goal is to choose $k$ such that the fraction of the total energy in the subspace

$$
\sum_{i=1}^{k} \lambda_{i} / \sum_{i=1}^{n} \lambda_{i}
$$

is close to one, yet $k$ is sufficiently small. Clearly this will not always be possible, with models which are better approximated by low-dimensional systems having relatively few large eigenvalues.

We now subtract the mean $\bar{x}$ from the dynamic equation, defining $e=x-\bar{x}$ to arrive at $\dot{e}(t)=f(e(t)+$ $\bar{x})$. We would like to approximate $e(t)$ in this equation by $P^{*} P e(t) \in S$.

Galerkin projection. The Galerkin projection has been used extensively to construct numerical solutions to partial differential equations. It can also be used to construct lower order mathematical models of a given dynamical system. The idea is to replace the given dynamics by an associated dynamics on a $k$-dimensional subspace $S \subset \mathbb{R}^{n}$ of the original state space, by projecting the vector field onto the tangent space of $S$. Using the coordinates defined previously, the resulting reduced order approximation is given by

$$
\dot{y}(t)=P f\left(P^{*} y(t)+\bar{x}\right)
$$

Clearly the subspace obtained by the KarhunenLoève method depends upon the inner product on the Euclidean space in which the state space is embedded; physical meaning is an important indicator of the appropriate choice of inner product.

Computational application of this method requires only standard matrix computations, despite its application to nonlinear systems. Also the method has the feature that it separates the model reduction into two parts; that of finding a suitable subspace and that of performing the projection. This lends physical intuition to the procedure, and allows alternative projection schemes to be used. 
We can expect such a procedure to work well for model reduction of the system within some given region of state space, and it is within such a specific region that data should be collected. In doing this, one must pay careful attention to symmetry, as in Glavaški [4] and references therein.

For control, a severe limitation is that the inputoutput behavior of the system is not taken into account. It is the generalization of the Karhunen-Loève reduction procedure to controlled systems that is the main focus of this paper, and we now proceed with this.

\section{Model reduction of controlled systems}

We now turn to the main problem addressed in this paper; that of model reducing nonlinear input-output systems of the form

$$
\begin{aligned}
\dot{x}(t) & =f(x(t), w(t)) \\
z(t) & =h(x(t))
\end{aligned}
$$

Here $x(t) \in \mathbb{R}^{n}$ is the state of the system, $w(t) \in \mathbb{R}^{p}$, and $z(t) \in \mathbb{R}^{q}$. The function $w$ is regarded as an input signal to the system, and the function $z$ as an output signal. The goal of model reduction is to construct another nonlinear system of differential equations

$$
\begin{aligned}
\dot{q}(t) & =\hat{f}(q(t), w(t)) \\
z(t) & =\hat{h}(q(t))
\end{aligned}
$$

where $q(t) \in \mathbb{R}^{k}$, and $k<n$, such that the input-output behavior of the two systems is similar, for states in some specific region of the the state space. That is, for a class of inputs $w$, we would like the outputs $z$ of the two systems to be similar. Before proceeding further, we need some preliminary definitions from the theory of linear systems.

\subsection{Linear systems}

The system (2) is called linear if it has a representation

$$
\begin{aligned}
& \dot{x}(t)=A x(t)+B w(t) \\
& z(t)=C x(t)
\end{aligned}
$$

where $x(t) \in \mathbb{R}^{n}$, and $A, B$, and $C$ are matrices of appropriate dimension. This representation is known as the state-space realization, and the system is described by the triple $(A, B, C)$. The linear system is called stable if the eigenvalues of $A$ all have real part strictly negative.

Suppose the system in equation (3) is stable. Then, for $u \in L_{2}(-\infty, 0]$ and denoting $u_{-}(t)=u(-t)$, the state at time zero $x(0)=x_{0}$ is given by

$$
x_{0}=\int_{0}^{\infty} e^{A s} B u_{-}(s) d s .
$$

This defines the controllability operator, $\mathcal{C}$ : $L_{2}[0, \infty) \rightarrow \mathbb{R}^{n}$ by $x_{0}=\mathcal{C} u_{-}$.

Lemma 2. Write $Y:=\mathcal{C C}^{*}$. Then $Y$ is the smallest semipositive solution to the Lyapunov equation

$$
A Y+Y A^{*}+B B^{*}=0 .
$$

The system is called controllable if $\operatorname{ImC}=\mathbb{R}^{n}$, in which case $Y>0$ and equation (4) has a unique solution. The matrix $Y$ is known as the controllability gramian.
We can define a similar notion for the output. Denote the future output by $y_{+}(t) \in L_{2}[0, \infty)$. Then, define the observability operator, $\mathcal{O}: \mathbb{R}^{n} \rightarrow L_{2}[0, \infty)$ by $y_{+}(t)=\mathcal{O} x_{0}$, and hence $\mathcal{O} x_{0}=C e^{A t} x_{0}$.

Lemma 3. Write $X:=\mathcal{O}^{*} \mathcal{O}$. Then $X$ is the smallest semipositive solution to the Lyapunov equation

$$
A^{*} X+X A+C^{*} C=0 .
$$

The system is said to be observable if $\operatorname{ker} \mathcal{O}=\{0\}$, in which case $X>0$ and equation (5) has a unique solution. The matrix $X$ is known as the observability gramian.

Both $X$ and $Y$ are $n \times n$ matrices, and they are given by the following integral formulae.

$$
\begin{aligned}
& Y=\int_{0}^{\infty} e^{A t} B B^{*} e^{A^{*} t} d t \\
& X=\int_{0}^{\infty} e^{A^{*} t} C^{*} C e^{A t} d t
\end{aligned}
$$

\subsection{Gramians and principal component analysis}

The method of principal component analysis relies on the use of data to construct the correlation matrix, with the underlying assumption that this data is collected from 'typical' system trajectories. We make the assumption that the initial state of the system is zero, and parametrize the trajectories with respect to the system input $u$.

For theoretical purposes, we are not restricted to samples, and can simply construct the correlation matrix using the integral

$$
R=\int_{0}^{\infty}(x(t)-\bar{x})(x(t)-\bar{x})^{*} d t
$$

where $x(t)$ is the state of the system at time $t$, and $\bar{x}$ is the mean state. We now apply a Karhunen-Loève decomposition to the states of a nonlinear input-output system of the form given by equation (2), parametrized by the inputs.

Define the following sets:

$$
\begin{aligned}
& \mathcal{T}^{n}=\left\{T_{1}, \ldots, T_{r} ; T_{i} \in \mathbb{R}^{n \times n}, T_{i}^{*} T_{i}=I, i=1, \ldots r\right\} \\
& \mathcal{M}=\left\{c_{1}, \ldots, c_{s} ; c_{i} \in \mathbb{R}, c_{i}>0, i=1, \ldots s\right\} \\
& \mathcal{E}^{n}=\left\{e_{1}, \ldots, e_{n} ; \text { standard unit vectors in } \mathbb{R}^{n}\right\}
\end{aligned}
$$

Here $\mathcal{T}$ is an arbitrary set of $r$ orthogonal matrices, and $\mathcal{M}$ is a set of $s$ positive constants. Given a function $u \in \ell_{\infty}$, define the mean $\bar{u}$ by

$$
\bar{u}:=\lim _{T \rightarrow \infty} \frac{1}{T} \int_{0}^{T} u(t) d t
$$

We make the standing assumptions that $x, z \in \ell_{\infty}$ and $z \in \ell_{2}$. For given initial conditions, these assumptions are satisfied for stable linear systems, and exponentially stable nonlinear systems, subject to a suitable definition of exponential stability.

Definition 4. Let $\mathcal{T}^{p}, \mathcal{E}^{p}$ and $\mathcal{M}$ be given sets as described above. For the system (2), define the empirical controllability gramian $\hat{Y}$ by

$$
\hat{Y}=\sum_{l=1}^{r} \sum_{m=1}^{s} \sum_{i=1}^{p} \frac{1}{r s c_{m}^{2}} \int_{0}^{\infty} \Phi^{i l m}(t) d t
$$


where $\Phi^{i l m}(t) \in \mathbb{R}$ is given by

$$
\Phi^{i l m}(t):=\left(x^{i l m}(t)-\bar{x}^{i l m}\right)\left(x^{i l m}(t)-\bar{x}^{i l m}\right)^{*}
$$

and $x^{i l m}(t)$ is the state of the system (2) corresponding to the impulsive input $w(t)=c_{m} T_{l} e_{i} \delta(t)$.

Lemma 5. For any nonempty sets $\mathcal{T}^{p}$ and $\mathcal{M}$, the empirical controllability gramian $\hat{Y}$ of the stable linear system $\dot{x}(t)=A x(t)+B w(t)$ is equal to the usual controllability gramian $Y$.

Proof. For the linear system,

$$
\begin{aligned}
\Phi^{i l m}(t) & =c_{m}^{2}\left(e^{A t} B T_{l} e_{i}\right)\left(e^{A t} B T_{l} e_{i}\right)^{*} \\
& =c_{m}^{2} e^{A t} B T_{l} e_{i} e_{i}^{*} T_{l}^{*} B^{*} e^{A^{*} t}
\end{aligned}
$$

hence

$$
\begin{aligned}
\hat{Y} & =\int_{0}^{\infty} \sum_{l=1}^{r} \sum_{m=1}^{s} \sum_{i=1}^{p} \frac{1}{r s} e^{A t} B T_{l} e_{i} e_{i}^{*} T_{l}^{*} B^{*} e^{A^{*} t} d t \\
& =\int_{0}^{\infty} \sum_{m=1}^{s} \frac{1}{s} e^{A t} B B^{*} e^{A^{*} t} d t=Y
\end{aligned}
$$

which is the desired result.

A variant of this result was first shown by Moore [9]; for impulsive inputs distributed in the above sense on the unit ball in $\mathbb{R}^{p}$, the Karhunen-Loève decomposition of the states leads to a construction of the controllability gramian for linear systems.

The empirical controllability gramian is a computable generalization of this to nonlinear systems; it has the property that the eigenvectors of $\hat{Y}$ corresponding to non-zero eigenvalues span a subspace $O \subset \mathbb{R}^{n}$ which contains the set of states reachable using the chosen initial impulsive inputs.

However, for controlled systems, simply studying the input-state behavior is not enough. The next definition is the analogue of the previous one for the output behavior.

Definition 6. Let $\mathcal{T}^{n}, \mathcal{E}^{n}$, and $\mathcal{M}$ be given sets as described above. For the system (2), define the empirical observability gramian $\hat{X}$ by

$$
\hat{X}=\sum_{l=1}^{r} \sum_{m=1}^{s} \frac{1}{r s c_{m}^{2}} \int_{0}^{\infty} T_{l} \Psi^{l m}(t) T_{l}^{*} d t
$$

where $\Psi^{l m}(t) \in \mathbb{R}^{n \times n}$ is given by

$$
\Psi_{i j}^{l m}(t):=\left(z^{i l m}(t)-\bar{z}^{i l m}\right)^{*}\left(z^{j l m}(t)-\bar{z}^{j l m}\right)
$$

and $z^{\text {ilm }}(t)$ is the output of the system (2) corresponding to the initial condition $x_{0}=c_{m} T_{l} e_{i}$.

Lemma 7. For any nonempty sets $\mathcal{T}^{n}$ and $\mathcal{M}$, the empirical observability gramian $\hat{X}$ of the stable linear system $\dot{x}(t)=A x(t)+B w(t), z(t)=C x(t)$ is equal to the usual observability gramian $X$.

Proof. For the linear system,

$$
\begin{aligned}
\Psi_{i j}^{l m}(t) & =c_{m}^{2}\left(C e^{A t} T_{l} e_{i}\right)^{*}\left(C e^{A t} T_{l} e_{j}\right) \\
& =c_{m}^{2} e_{i}^{*} T_{l}^{*} e^{A^{*} t} C^{*} C e^{A t} T_{l} e_{j}
\end{aligned}
$$

hence

$$
\Psi^{l m}(t)=c_{m}^{2} T_{l}^{*} e^{A^{*} t} C^{*} C e^{A t} T_{l}
$$

and

$$
\hat{X}=\sum_{m=1}^{s} \frac{1}{s} \int_{0}^{\infty} e^{A^{*} t} C^{*} C e^{A t} d t=X
$$

which is the desired result.

We now have the tools we need for empirical analysis of the input-output behavior of the nonlinear system. Rather than searching for exact controllability and observability submanifolds within the state space, our approach is to search for subspaces which approximate these manifolds. The advantage of this approach lies in the computation; all that is required is the solution of standard linear matrix eigenvalue problems.

Unlike in the autonomous case, we now have two important subspaces of the state space, and their corresponding eigenvalues. We can proceed in the same manner as for linear systems, and make use of the ideas of balanced realization theory to decide on which subspace to project. We therefore return to a description of linear systems theory.

\subsection{Model reduction via balanced truncation}

In this section we give a brief overview of the method of balanced truncation.

The Hankel operator of a linear system is the operator which maps (the reflection of) past inputs to future outputs, assuming the future input is zero. Denoting as before $u_{-}(t)=u(-t)$, the Hankel operator, $\Gamma$, is given by the map $\Gamma:=\mathcal{O C}$ so that $y_{+}=\Gamma u_{-}$.

Since $x_{0}=\mathcal{C} u_{-}$and $y_{+}=\mathcal{O} x_{0}$, it is immediate that the Hankel operator has at most rank $n$, equal to the state space dimension of the system. A singular value decomposition of this linear operator leads to $n$ singular values, known as the Hankel singular values, which are independent of the particular state space realization of the system, and are a property of the system as an input-output map.

The Hankel singular values are the eigenvalues of $\Gamma \Gamma^{*}$. These are the eigenvalues of $\mathcal{O C C}^{*} \mathcal{O}^{*}$, which are the same as those of the $n \times n$ matrix $\mathcal{O}^{*} \mathcal{O C C}^{*}=X Y$. The balanced realization gives a way to find which particular states correspond to which Hankel singular value.

Definition 8. Suppose $A$ is stable. The realization $(A, B, C)$ is called balanced if the controllability gramian $Y$ and the observability gramian $X$ are equal and diagonal.

We assume throughout the following that the system $(A, B, C)$ is stable, controllable, and observable. In this case, there will always exist a nonsingular state transformation $T$ such that the new realization $\left(T A T^{-1}, T B, C T^{-1}\right)$ is balanced.

This leads to a method of model reduction known as balanced truncation, introduced by Moore [9] in the context of realization theory. The procedure is to truncate those states from the balanced realization corresponding to small Hankel singular values $\sigma_{i}$. If the states are ordered according to decreasing singular 
value, this is equivalent to applying a Galerkin projection to the balanced realization, where $P=\left[\begin{array}{ll}I & 0\end{array}\right]$.

If $(A, B, C)$ is balanced, the truncated system is given by

$$
\begin{aligned}
& \dot{y}(t)=P A P^{*} y(t)+P B u(t) \\
& z(t)=C P^{*} y(t)
\end{aligned}
$$

The reduced-order model constructed via balanced truncation is independent of the original state space realization, and has several useful properties. In particular the truncated system is stable if the truncation does not 'split' states corresponding to a $\sigma_{i}$ with a multiplicity greater than one. A-priori error bounds in the induced 2-norm are known for the error between the original and the reduced system; these were first shown independently by Enns [3] and Glover [5].

\subsection{Empirical balanced truncation}

The empirical gramians give a precise method for deciding upon the importance of particular subspaces of the state space, with respect to the inputs and outputs of the system.

We propose to use these for model reduction in the same way as for linear systems; find a linear change of coordinates such that the empirical gramians are balanced, and perform a Galerkin projection onto the states corresponding to the largest eigenvalues of $\hat{X} \hat{Y}$.

Since for linear systems, the empirical gramians are exactly the usual gramians, this method is exactly balanced truncation when it is applied to a linear system. When applied to a nonlinear system, it requires only matrix computations, and results in a new nonlinear model.

Let $T$ be the change of coordinates such that the system is balanced; that is $T Y T^{*}=T^{-1 *} X T^{-1}=\Sigma$, and let $P=\left[\begin{array}{ll}I & 0\end{array}\right]$ be the $k \times n$ projection matrix. Applying the previous analysis leads to a reduced order model given by

$$
\begin{aligned}
& \dot{y}(t)=P T f\left(T^{-1} P^{*} y(t), w(t)\right) \\
& z(t)=h\left(T^{-1} P^{*} y(t)\right)
\end{aligned}
$$

This is the final reduced order model. This empirical balanced truncation gives a reduced order model which takes into account the input-output behavior, and is directly computable from data.

\section{Computation}

We can directly apply Definitions 4 and 6 for construction of the empirical gramians from data, for nonlinear systems. The data may be taken either from simulation or from experiment. All that is needed is numerical approximation of the above integrals, using sampled-data from both $x(t)$ and $z(t)$. We can take advantage of knowledge of $f$ to calculate $\dot{x}(t)$ in this computation to achieve higher order accuracy, and increase the allowable sample time.

To perform the required experiments, it is necessary to be able to set the initial state of the system, to measure the state at all times, and to apply approximately impulsive inputs. These conditions may be satisfied for certain mechanical systems, although in the case of fluid dynamics, for example, we are forced to rely on data from simulation.
We also need to choose the sets $\mathcal{T}$ and $\mathcal{M}$. A reasonable simple choice is $\mathcal{T}=\{I,-I\}$, since this corresponds to using both positive and negative inputs (or initial states) on each input separately. For distributed actuators, larger sets may be justified.

The sets $\mathcal{M}$ specify the size of inputs and states we are interested in. The choice is motivated by the magnitudes of the inputs and states actually seen in experiments.

As for autonomous systems, a crucial feature of the Karhunen-Loève procedure is that in general we should expect it to work well only in limited regions of state space. We do not expect to be able to approximate global behavior of the system well using a linear projection; however, it is perhaps more reasonable to expect to be able to construct low order models for systems within some given operating region.

A simple technique for balancing the empirical gramians $\hat{X}$ and $\hat{Y}$ is as follows. First, construct a Cholesky factorization of $\hat{Y}$ so that $\hat{Y}=Z Z^{*}$. Let $U \Sigma^{2} U^{*}$ be a singular value decomposition of $Z^{*} \hat{X} Z$, and let $T=\Sigma^{\frac{1}{2}} U^{*} Z^{-1}$. Then $T \hat{Y} T^{*}=\Sigma$, and $T^{-1 *} \hat{X} T^{-1}=\Sigma$, as desired.

We can now change state coordinates of the nonlinear system, and truncate using the Galerkin projection. The rows of $T$ give the modes of the system.

\section{Mechanical links example}

Not only are high-order systems hard to control, but it is difficult to develop intuition as to their behavior. One of the advantages of the procedure developed here is that it can be viewed as a selection of appropriate nonlinear mode shapes on which to project the dynamics. These mode shapes often are physically meaningful, and in this section we give examples for a simple, mildly nonlinear mechanical system.

The system considered here consists of five rigid rods, connected via torsional springs and dampers in the plane. The lowest rod is pinned to ground with a torsional spring, so that the system has a stable equilibrium in the upright vertical position.

The system has a single input, a torque about the lowest pin joint, and a single output, the horizontal displacement of the end of the last rod from the vertical symmetry axis.

\subsection{Linearized model}

We first analyze the linearization of the system about its stable equilibrium; Figure 1 shows the first four of the ten mode shapes and the corresponding singular values of the balanced realization. The singular values have been normalized so that they sum to one.

\subsection{Nonlinear Lagrangian model}

Application of the above empirical model reduction procedure to the nonlinear Lagrangian model of this system leads to a set of corresponding modes. For a particular choices of the magnitude constants $c_{i}$, Figure 2 shows these modes; we can see that the first two mode shapes have split into three.

Figure 3 shows the mode shapes at an increased value for the magnitude constants; the mode shapes become significantly different from those of the linearization. 


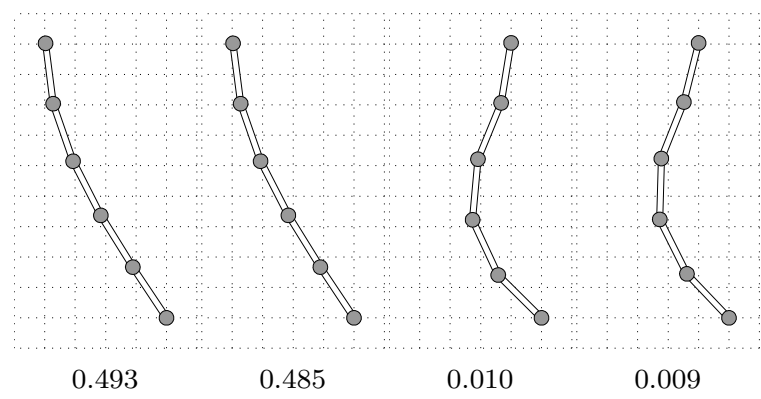

Figure 1: The balanced modes of the linearization, with the corresponding fraction of energy in each mode.

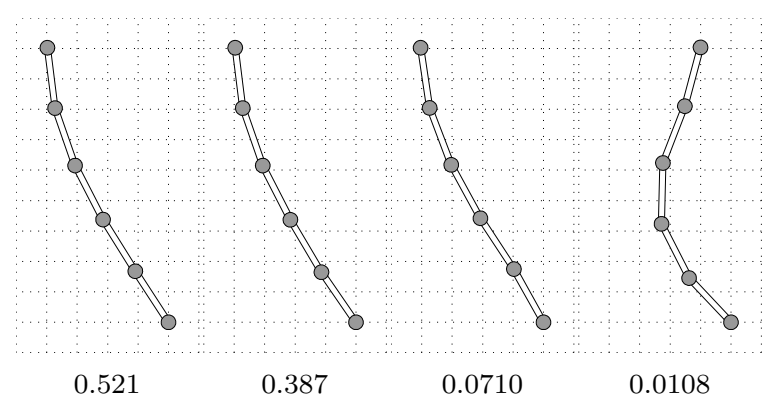

Figure 2: The empirical balanced modes of the nonlinear model, for small inputs.

\section{Conclusions}

In this paper we have presented new methods for model reduction of nonlinear systems. These methods combine the physical intuition of Karhunen-Loève techniques, as they have been applied to analysis of complex flows, with the control engineering methodology of balanced truncation.

The resulting method of model reduction is based on data. It requires only simple matrix computations, and may be applied directly to nonlinear systems. When applied to linear systems, the reduced order model constructed is exactly the usual balanced truncation of the system.

The choice of impulsive inputs, and initial states distributed uniformly on the unit sphere, are perhaps somewhat arbitrary methods of parametrizing trajectories for nonlinear systems. Many other methods might be suggested, and for control a very reasonable strategy would be to apply a Karhunen-Loève decomposition of the states seen during simulation of closedloop behavior. There are several other possibilities

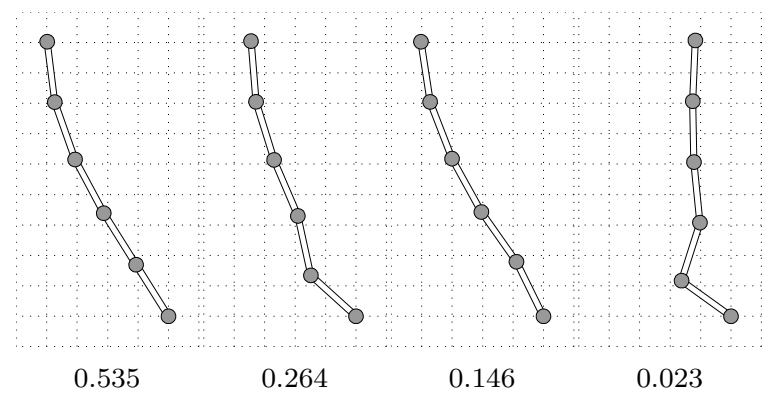

Figure 3: The empirical balanced modes of the nonlinear model, for large inputs. that might be suggested; our focus here has been the construction of a computable extension of balanced truncation for nonlinear systems.

For mechanical systems, a better approach is to perform the Karhunen-Loève expansion on the configuration space rather than the phase space, preserving the mechanical structure of the system in the reduction [7]. Similarly, taking account of symmetry [4] and other special structure present in the original system can be of great use, leading to reduced computational requirements and producing more accurate reducedorder models.

One advantage of the empirical procedure described in this paper is that often the simulation itself has been implemented using known heuristics; an example is in fluid dynamics, where often vortex methods are used to implement simulations of incompressible flow. Even though the simulation is providing a numerical solution to a different set of equations, the reconstructed flow field from the simulation may be used to produced a reduced-order approximation to the equations of potential flow. This gives a method for taking advantage of known heuristics about the behavior of such systems, which are often used in simulation but difficult to incorporate into control design.

\section{References}

[1] G. Berkooz, P. Holmes, and J. L. Lumley. The proper orthogonal decomposition in the analysis of turbulent flows. Annual Review of Fluid Mechanics, 25:539-575, 1993.

[2] A. M. Bloch. Estimation, principal components, and Hamiltonian systems. Systems and Control Letters, 6:103-108, 1985.

[3] D. F. Enns. Model reduction for control system design. PhD thesis, Stanford university, 1984.

[4] S. Glavaški, J. E. Marsden, and R. M. Murray. Model reduction, centering, and the KarhunenLoève expansion. IEEE Conference on Decision and Control, 1998.

[5] K. Glover. All optimal Hankel-norm approximations of linear multivariable systems and their Linfinity error bounds. International Journal of Control, 39:1115-1193, 1984.

[6] P. Holmes, J. L. Lumley, and G. Berkooz. Turbulence, coherent structures, dynamical systems, and symmetry. Cambridge University Press, 1996.

[7] S. Lall and J. E. Marsden. Model reduction of mechanical systems. IGPP/NPACI Workshop on Accurate Simulation and Modeling of Physical Systems, 1998.

[8] J. L. Lumley. The structure of inhomogeneous turbulence. In A. M. Yaglom and V. I. Tatarski, editors, Atmospheric turbulence and wave propagation, pages 166-178. Nauka, Moscow, 1967.

[9] B. C. Moore. Principal component analysis in linear systems: controllability, observability, and model reduction. IEEE Transactions on Automatic Control, 26(1):17-32, February 1981.

[10] K. Pearson. On lines and planes of closest fit to points in space. Philosophical magazine, 2:609629, 1901.

[11] J. M. A. Scherpen. H-infinity balancing for non- 
linear systems. International Journal of Robust and Nonlinear Control, 6:645-668, 1996. 